\title{
Advanced magnetic resonance imaging of cortical laminar necrosis in patients with stroke
}

\author{
Roberto Cannella ${ }^{1}\left(\mathbb{D}\right.$, Gianvincenzo Sparacia ${ }^{1,2}\left(\mathbb{D}\right.$, Vincenzina Lo $\mathbf{R e}^{3}$, \\ Elisa $\mathbf{O d d o}^{1}$, Giuseppe Mamone ${ }^{2}$ and Roberto Miraglia ${ }^{2}$
}

(SSAGE

\begin{abstract}
Purpose: The aim of this study was to assess the novel advanced magnetic resonance imaging findings of acute stage cortical laminar necrosis developing after complicated cardiovascular or abdominal surgery.

Materials and methods: This institutional review board-approved study included patients with postoperative stroke due to cortical laminar necrosis imaged with magnetic resonance in the acute stage. Brain magnetic resonance imaging examinations were obtained on a $3 T$ magnetic resonance scanner within 48 hours of the neurological symptoms, including diffusion-weighted images (b value, $1000 \mathrm{~s} / \mathrm{mm}^{2}$ ) and arterial spin labelling using a pseudo-continuous arterial spin labelling method in four patients. Conventional and advanced magnetic resonance images were analysed to assess the imaging features in acute stage cortical laminar necrosis.

Results: The final population consisted of 14 patients (seven men and seven women, mean age 61 years, range 32-79 years) diagnosed with stroke and acute phase cortical laminar necrosis. All the patients presented with cortical lesions showing restricted diffusion on diffusion-weighted images and hypointensity on the apparent diffusion coefficient map. Cortical hyperintensity on T2-weighted or fluid-attenuated inversion recovery images was found in three (21\%) and six (43\%) patients, respectively. Reduced perfusion was noted in three out of four patients imaged with arterial spin labelling, while in one case no corresponding perfusion abnormality was noted on the arterial spin labelling maps. Arterial spin labelling abnormalities were much more extensive than diffusion restriction in two patients, and they were associated with a poor outcome.

Conclusion: Cortical hyperintense abnormalities on diffusion-weighted imaging may be the only sign of developing cortical laminar necrosis injury. The acquisition of arterial spin labelling helps to identify perfusion alterations and the extension of the ischaemic injury.
\end{abstract}

\section{Keywords}

Cortical laminar necrosis, stroke, magnetic resonance imaging, diffusion-weighted imaging, arterial spin labelling

\section{Introduction}

Cortical laminar necrosis (CLN) is a permanent ischaemic injury characterised by damage of selective cortical layers. Within the cerebral cortex, the third layer is the most vulnerable, followed by the fifth or sixth, with the second and fourth being relatively more resistant to ischaemic necrosis from hypoperfusion. ${ }^{1}$ CLN usually occurs when the degree of hypoxic injury is not enough to cause complete cortical damage. The white matter, which is less metabolically active, is usually not involved in this manifestation of ischaemia.

Various clinical conditions have been associated with the development of CLN. This is most commonly secondary to anoxic or hypoxic-ischaemic encephalopathy following a cardiac and/or respiratory arrest, with consequent reduction of oxygenation and glucose supply to the neurons. ${ }^{1}$ CLN may also manifest in conditions with increased glucose and oxygen request of the brain, as in status epilepticus when hyperperfusion is not sufficient to supply the hyperactive and hypermetabolic cortical area. ${ }^{2}$ Manifestations of CLN have also been reported in migrainous infarction, moyamoya

${ }^{1}$ Radiology Service, Biomedicina, Neuroscienze e Diagnostica avanzata (BIND) Department, University of Palermo, Italy

${ }^{2}$ Radiology Service, Department of Diagnostic and Therapeutic Services, Mediterranean Institute for Transplantation and Advanced Specialized Therapies (IRCCS-ISMETT), Italy

${ }^{3}$ Neurology Service, Department of Diagnostic and Therapeutic Services, Mediterranean Institute for Transplantation and Advanced Specialized Therapies (IRCCS-ISMETT), Via Ernesto Tricomi 5, 90127, Palermo, Italy

\section{Corresponding author:}

Gianvincenzo Sparacia, Radiology Service, Biomedicina, Neuroscienze e Diagnostica avanzata (BIND) Department, University of Palermo, Via del Vespro 127, 90127 - Palermo, Italy; Department of Diagnostic and Therapeutic Services UPMC IRCCS-ISMETT, (Mediterranean Institute for Transplantation and Advanced Specialized Therapies), Palermo, Via Ernesto Tricomi 5, 90127 Palermo, Italy.

Email: gianvincenzo.sparacia@unipa.it 
disease, intoxication, hypoglycemia, metabolic disorders, immunosuppressive treatment and chemotherapy. $^{3-8}$

Magnetic resonance imaging (MRI) represents the most accurate method for the non-invasive diagnosis of CLN. CLN lesions are particularly challenging to diagnose in the acute stage using only clinical neurological symptoms and conventional MRI. The typical hyperintense cortical signal lesion on T1-weighted images usually appears only 2 weeks after the clinical onset during the subacute or chronic phases., 1,9 Although the brain may appear normal on conventional MRI in the early acute phase, the detection of acute CLN has significant clinical implications due to the higher risk of neurological complications and permanent brain damage if untreated. ${ }^{2}$ Only a few case reports have assessed the abnormality on diffusionweighted imaging (DWI) in acute phase CLN. ${ }^{3,4,10,11}$ Thus this entity still has several unexplored characteristics and there is a significant gap in the knowledge regarding the acute phase of CLN, specially with advanced MRI techniques.

Advanced MRI technique availability is increasing in clinical practice. DWI is routinely performed for the early detection of both local and global cerebral infarction, with the hyperintensity on DWI reflecting the restricted diffusion of free water due to cytotoxic oedema. Perfusion imaging represents a new frontier for advanced MRI studies and is an area of continuous investigation. Among the different perfusion techniques, arterial spin labelling (ASL) allows the noninvasive measurement of cerebral perfusion through magnetically labelled arterial blood water protons as an endogenous tracer. ${ }^{12}$ We hypothesise that advanced MRI may play a significant role in the diagnosis of CLN lesions in the acute phase, and may help to redefine the extension through the evaluation of the perfusion-diffusion mismatch of the lesion even in critical patients after major cardiovascular or abdominal surgical procedures. Therefore, the purpose of this study was to explore the added diagnostic value of arterial spin labeling technique in patients with cortical laminar necrosis in acute phase of stroke.

\section{Materials and methods}

Our retrospective cohort study was reviewed and approved by the institutional research review board of our institution, and informed consent was waived; however, informed written consent to the MRI was obtained in all patients.

\section{Patient population}

Medical and radiological records of patients referred at our institution, a tertiary referring centre for transplantation and cardiothoracic surgery, between January 2017 and December 2018 were reviewed by two of the authors (EO and RC, with 6 and 5 years of experience in neuroimaging, respectively) uninvolved in the imaging interpretation. The initial population consisted of 435 patients undergoing brain MRI using the same protocol with the exception of ASL images and neurological evaluation in the perioperative period with suspected complications. Among them, patients were selected if they matched the following inclusion criteria: (a) age 18 years or older; (b) postoperative stroke occurring after major cardiovascular of abdominal surgery; (c) MRI within 48 hours after the onset of neurological symptoms; (d) the presence of ischaemic lesions involving the cerebral cortex without involvement of the adjacent white matter, suggestive of CLN. ${ }^{9}$ Clinical data, including the type of surgical procedure, neurological symptoms and outcome, were collected for each patient.

\section{MRI technique and imaging analysis}

Brain MRI examinations were obtained on a $3 \mathrm{~T}$ MR scanner (Discovery 750w; General Electric Healthcare, Milwaukee, WI, USA) with a 32 channel brain coil. The MRI protocol included axial and sagittal fastspin echo (FSE) T2W (8000/90 (TR/TE)) images, axial fluid-attenuated inversion recovery (FLAIR) (9000/150/2250 (TR/TE/TI)) images, along with axial, sagittal and coronal non-enhanced FSE T1W (600/20 (TR/TE)) images with a field of view (FOV) of $24 \mathrm{~cm}$, matrix $320 \times 320$, slice thickness $4 \mathrm{~mm}$, intersection gap $1 \mathrm{~mm}$, number of excitations 1 . DWI magnetic resonance images were acquired with the following parameters: repetition time (TR), $6000 \mathrm{~ms}$; echo time (TE), $88 \mathrm{~ms}$; b value, $1000 \mathrm{~s} / \mathrm{mm}^{2}$; image averaging, two times; FOV $230 \mathrm{~mm}$.

The ASL sequence was prepared using a three-dimensional, spiral, FSE sequence with background suppression for perfusion imaging covering the entire brain. A pseudo-continuous arterial spin labelling (PCASL) method $^{12}$ was used with the following parameters: eight arms with 512 points in each spiral arm, phase encoding in the $z$ direction: 32 , slice thickness: $2.5 \mathrm{~mm}$, slice spacing $2.5 \mathrm{~mm}$, TR: 5301, echo time TE: 12 , echo train length (ETL): 1, post-label delay: 2025 seconds, number of excitations: 2, FOV: $24 \mathrm{~cm}$. Cerebrovascular perfusion maps were processed from ASL images on a dedicated workstation (Advantage Workstation version 4.7; General Electric Healthcare, Milwaukee, WI, USA). ASL images were obtained at the discretion of the interpreting radiologist based on patients' clinical characteristics and scanning time in the initial inclusion time for this study with progressive implementation in clinical practice, while it was included as part of the standard brain MRI protocol from June 2018, and acquired in all subsequent patients undergoing MRI for suspected stroke.

The brain magnetic resonance images were analysed in consensus by two radiologists (GS and GM, with 29 and 15 years of experience in neuroimaging, respectively) who were unaware of the patients' clinical 
information. The radiologists evaluated the localisation of CLN lesions in the cerebral cortex and the signal intensity on T1-weighted, T2-weighted and FLAIR images, along with susceptibility-weighted imaging (SWI) sequences and the presence of restricted diffusion on DWI and apparent diffusion coefficient (ADC) maps. Lesions were scored as hypointense, isointense or hyperintense on each sequence. The distribution of hypoperfusion areas on ASL was also recorded, as well as the presence of diffusion-perfusion mismatch.

\section{Results}

\section{Patient characteristics and conventional MRI findings}

The characteristics of the included patients and MRI findings in the acute stage are reported in Table 1 . The final population consisted of 14 patients, which were seven men and seven women, with a mean age of $61.1 \pm 13.3$ years, range $23-79$ years.

In all patients, MRI was performed within 48 hours form the onset of acute neurological deficit after major cardiac $(n=6,43 \%)$ or abdominal $(n=8,57 \%)$ surgical procedures. Clinical neurological manifestations in these patients included epilepsy, rapidly developing coma, dysarthria or hemiparesis. On MRI, all the patients were characterised by cortical lesions showing restricted diffusion on DWI images and hypointensity on the ADC map. The ischaemic lesions were typically confined in the cerebral cortex, without involvement of the adjacent white matter. The lesions were distributed in the frontal lobe in the majority of patients $(n=11$, $79 \%)$, followed by the parietal lobe $(n=6,43 \%)$ and then the temporal or occipital lobe $(n=5,36 \%$ in both lobes). Six out of $14(43 \%)$ patients presented with bilateral ischaemic lesions involving more than one cerebral lobe (Figure 1).

Regarding conventional MRI sequences, no signal abnormalities were observed in the acute stage on T1-weighted images or SWI sequences in any patient. Hyperintense signal intensity was noted on T2-weighted or FLAIR images on three $(21 \%)$ and six $(43 \%)$ patients, respectively. In contrast, cortical restricted diffusion on DWI images was the only abnormality detected in eight $(57 \%)$ patients, without correlation in other sequences.

\section{Arterial spin labelling MRI}

Four out of 14 patients presenting with conventional MRI evidence of acute stage CLN were imaged with ASL in the acute phase. In two patients there was a significant mismatch between the extension of signal abnormalities on DWI and the hypoperfusion noted on ASL maps. In particular, in the first case DWI images demonstrated multiple bilateral regions with restricted diffusion and hypointensity on the ADC map at the level of the cortex showing laminar
Table 1. Characteristics of the included patients and MRI findings of cortical lesions in the acute stage.

\begin{tabular}{|c|c|}
\hline Characteristics & Number (\%) \\
\hline Patients & $14(100)$ \\
\hline \multicolumn{2}{|l|}{ Gender } \\
\hline Men & $7(50)$ \\
\hline Women & $7(50)$ \\
\hline Age (years) & $\begin{array}{l}61.1 \pm 13.3 \\
\quad(\text { range } 32-79)\end{array}$ \\
\hline \multicolumn{2}{|l|}{ Surgical procedure } \\
\hline Cardiovascular & $6(43)$ \\
\hline Abdominal & $8(57)$ \\
\hline \multicolumn{2}{|l|}{ Ischaemic localisation } \\
\hline Frontal lobe & $11(79)$ \\
\hline Parietal lobe & $6(43)$ \\
\hline Temporal lobe & $5(36)$ \\
\hline Occipital lobe & $5(36)$ \\
\hline Bilateral & $6(43)$ \\
\hline \multicolumn{2}{|l|}{ T1-weighted sequence } \\
\hline Hyperintense & $0(0)$ \\
\hline Isointense & $14(100)$ \\
\hline Hypointense & $0(0)$ \\
\hline \multicolumn{2}{|l|}{ T2-weighted sequence } \\
\hline Hyperintense & $3(21)$ \\
\hline Isointense & $11(79)$ \\
\hline Hypointense & $0(0)$ \\
\hline \multicolumn{2}{|l|}{ FLAIR } \\
\hline Hyperintense & $6(43)$ \\
\hline Isointense & $8(57)$ \\
\hline Hypointense & $0(0)$ \\
\hline \multicolumn{2}{|l|}{ SWI } \\
\hline Hypointense & $0(0)$ \\
\hline Iso- to Hyperintense & $14(100)$ \\
\hline \multicolumn{2}{|l|}{ DWI } \\
\hline Diffusion restriction present & $14(100)$ \\
\hline Diffusion restriction absent & $0(0)$ \\
\hline
\end{tabular}

MRI: magnetic resonance imaging; FLAIR: fluid-attenuated inversion recovery; SWI: susceptibility-weighted imaging; DWI: diffusion-weighted imaging.

distribution, localised on the temporal, occipital and frontal lobes, respectively. The ASL maps showed much more extensive damage, with larger cortical areas of hypoperfusion in the right temporal lobe (Figure 2). Similarly, the second case demonstrated multiple cortical areas of laminar restricted diffusion in the right temporal, occipital and parietal lobes, while hypoperfusion was also noted in the contralateral areas when using ASL imaging. In both cases, the patients died within 7 days after the neurological onset due to the progression of severe cortical damage, despite intensive care treatment.

In the remaining two cases imaged with ASL there was either a substantial overlapping between the areas of restricted diffusion involving the cerebral cortex and 


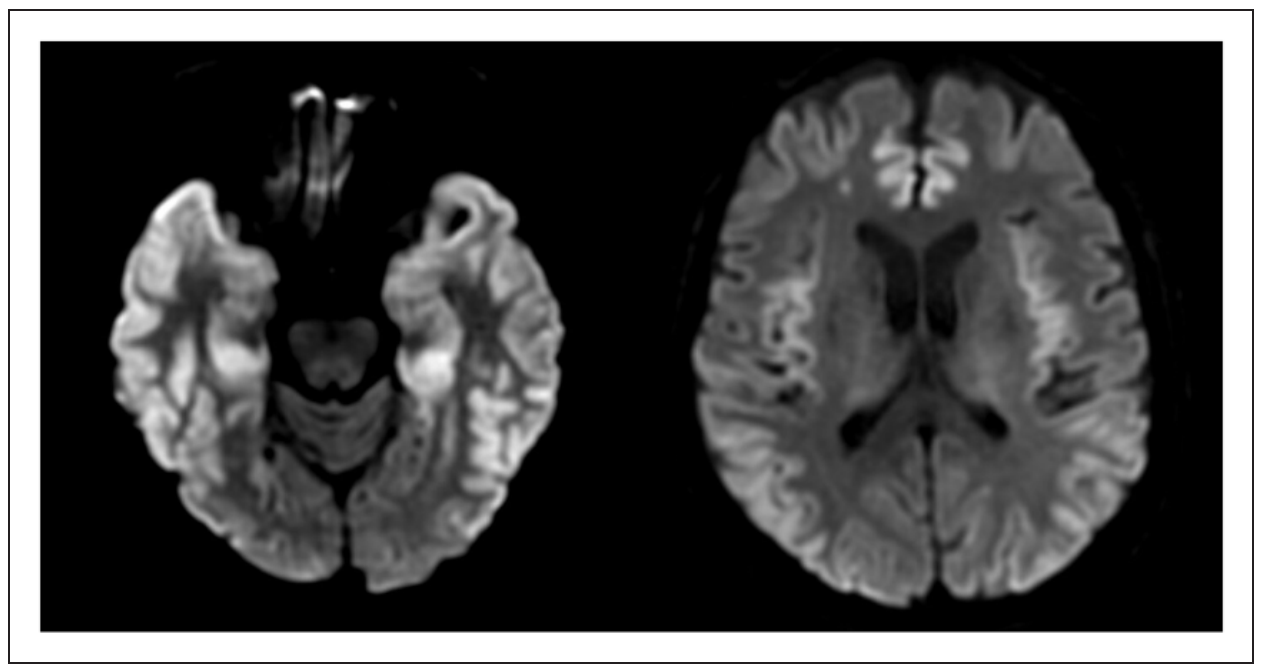

Figure 1. A 68-year-old man with acute-phase cortical laminar necrosis after cardiac surgery. Magnetic resonance images on diffusionweighted imaging show diffuse bilateral cortical areas with restricted diffusion in the temporal, frontal and parietal lobes, which represented the first sign of the development of cortical laminar necrosis.

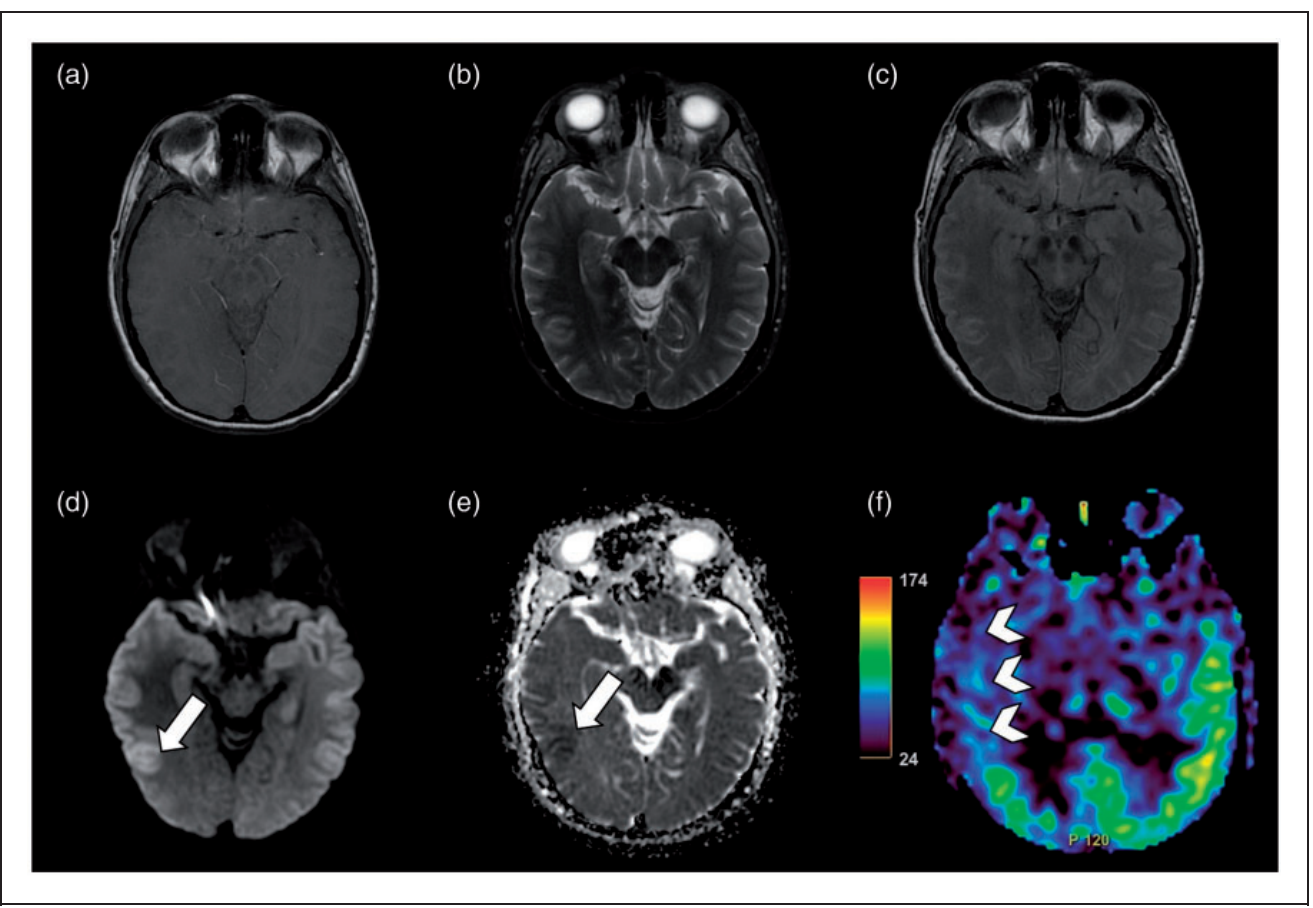

Figure 2. A 41-year-old woman with acute phase cortical laminar necrosis after orthotopic liver transplantation. Magnetic resonance imaging acquired on axial (a) T1-weighted, (b) T2-weighted, (c) FLAIR, (d) diffusion-weighted imaging, (e) ADC map and (f) ASL map. Cortical gyriform lesions are demonstrated in the right temporal lobe showing restricted diffusion and hypointensity on the ADC map, consistent with cortical laminar necrosis. A significantly larger area of hypoperfusion is noted on corresponding ASL images (arrows) involving all the right temporal lobe, with significantly reduced perfusion compared to the contralateral left temporal lobe. FLAIR: fluidattenuated inversion recovery; ADC: apparent diffusion coefficient; ASL: arterial spin labelling.

the hypoperfusion detected on ASL images, or no abnormal perfusion noted on the ASL maps in the corresponding areas with diffusion restriction in a patient with MRI acquired the day after the onset of the stroke. After the resolution of the acute ischaemic symptoms, both patients were discharged and planned for neuromotor rehabilitation.

\section{Discussion}

We present imaging findings of 14 patients with acute phase CLN with stroke occurring in the postoperative period as a complication of major surgical cardiovascular or abdominal surgical procedures, including the novel advanced MRI appearance of four patients 
imaged with ASL as advanced MRI. To the best of our knowledge this is the first study of CLN imaged with both DWI and ASL imaging.

In our population the lesions were distributed in the cerebral cortex, with gyriform pattern and sparing of the subcortical white matter, which are characteristics compatible with the radiological diagnosis of CLN. ${ }^{13}$ While no signal abnormalities were detected in the acute phase on T1-weighted or SWI sequences, T2-weighted and FLAIR images already showed hyperintense cortical lesions without involvement of the adjacent white matter in $21 \%$ and $43 \%$ of patients, respectively. These findings are concordant with prior studies reporting laminar cortical hyperintensity on T1-weighted imaging visible only 2 weeks after the initial clinical manifestations ${ }^{14}$ and no significant abnormalities on additional T2-weighted gradient echo or SWI sequences that could reflect the presence of paramagnetic substances such as haemosiderin or calcifications. 9,15,16 Radiological-pathological correlation performed with autopsies was obtained in previously published studies only in a few cases in patients with CLN, and described correlation with the T1 shortening on subacute/chronic phase MRI with the presence of necrotic debris throughout the cortex with fat-laden macrophages, but without evidence of haemorrhages or calcifications. ${ }^{16-18}$

Diffusion restriction was the only sign present in all our patients with acute phase CLN, and was observed even without signal abnormalities on the other conventional sequences in $57 \%$ of patients. Only a few previous case reports have described the appearance of CLN in the acute phase including DWI imaging. ${ }^{3,4,10,11}$ Lee et al. ${ }^{3}$ reported a case of CLN due to hypoglycemia appearing as cortical lesions with restricted diffusion and gyriform distribution on MRI performed after 2 days of the clinical onset. Choi et al. ${ }^{4}$ described the diffusion restriction preceding the appearance of CLN in a patient with acute hepatic encephalopathy. This evidence suggests that the diffusion restriction may be present even without any additional correlation on other sequences in the acute phase. The DWI abnormalities are usually reversible after 2-4 weeks and they were associated with poor outcome in previous series. $^{3,4,10,11,19}$

Advanced MRI techniques including ASL were performed in four patients in our study. Hypoperfusion areas were noted in three out of four patients imaged with ASL. In contrast, in one of our cases no corresponding perfusion abnormality was noted on the ASL maps. This could be explained by the timing of the MRI examination acquired at more than 24 hours after the clinical onset, while in the remaining three cases the MRI examinations were performed in the first 12 hours. In one patient there was a significant overlapping between the location of DWI restriction and the areas of altered decreased perfusion relieved on ASL maps. Interestingly, in two of our cases the ASL abnormalities were much more extensive than diffusion restriction, and the prognosis of these patients was particularly poor, with death within 7 days of intensive care. Further studies with a larger number of patients will be necessary to explore the prognostic value of ASL in CLN, and evaluate the mismatch with diffusion restriction. ${ }^{20}$ Abnormal hypoperfusion on MRI has been reported only in the subacute/chronic phase by Morais et al. ${ }^{21}$ in a recently published case of migrainous infarction presenting as CLN. The MRI examination performed one month after the migraine attack showed a cortical-based gyriform lesion, with hyperintensity on T1-weighted imaging and cortical enhancement after contrast administration associated with hypoperfusion on perfusion-weighted imaging (PWI) and the absence of activation of the injured cortex on functional MRI.

ASL represents a novel advanced MRI technique that allows the non-invasive measurement of the cerebral perfusion without contrast injection. ${ }^{2-25}$ Compared to other imaging technique for the evaluation of brain perfusion (PWI), the major advantages of ASL are the non-invasiveness, the repeatability over time and the possibility to obtain information about perfusion without the need for intravenous gadolinium contrast administration. ${ }^{26}$ The ASL may be integrated in the standard MRI protocol for the evaluation of ischaemic patients with minimal increment of scanning time. This may be particularly helpful in critical patients with hypoperfusion complex and multi-organ failure, which are often accompanied by transient acute renal injury, contraindicating intravenous contrast administration. ${ }^{26}$ Promising clinical applications of ASL are emerging for the evaluation of Alzheimer's disease and dementia, arteriovenous malformation, epilepsy, tumour histology and progression, and finally acute or chronic perfusion abnormality. ${ }^{23,24,26-28}$ In our initial experience, ASL was adequately performed even in critical patients in the postoperative period, allowing us to obtain additional information regarding the perfusion abnormalities in the acute phase of ischaemic lesions. The lack of contrast administration is the major advantage of ASL as opposed to other perfusion techniques (PWI), especially for critical patients with hypoperfusion complex associated with possible acute kidney failure. We speculated that perfusion abnormalities demonstrated on the ASL maps in the acute phase in our series may be explained by the presence of reduced blood supply in the setting of nonocclusive hypoxic-ischaemic encephalopathy. ${ }^{24,29-31}$

Finally, we acknowledge the several limitations of this study, most notably related to the retrospective study design and to the small number of patients included. However, the latter reflects the rarity of CLN in clinical practice, especially in the acute phase in the postoperative setting. We did not have a control group to compare the MRI findings on DWI and ASL images. Furthermore, the lack of long-term imaging follow-up may have limited the imaging analysis of the evolution of CLN lesions. 
In conclusion, we presented the imaging appearance of CLN in the acute phase, imaged with both conventional and advanced MRI techniques. Cortical hyperintense abnormalities on DWI may be the only sign of developing CLN injury, even without correlation on other sequences. The acquisition of ASL may help to identify perfusion alterations and the extension of the ischaemic injury and likely correlates with a poor prognosis. A further prospective study, including a larger number of patients, may be helpful to understand better the chronological changes and outcome of CLN.

\section{Conflict of interest}

The authors declared no potential conflicts of interest with respect to the research, authorship, and/or publication of this article.

\section{Funding}

This research received no specific grant from any funding agency in the public, commercial, or not-for-profit sectors.

\section{Informed consent}

Our retrospective cohort study was reviewed and approved by the institutional research review board of our institution, and informed consent was waived; however, informed written consent to the MRI was obtained in all patients.

\section{ORCID iD}

Roberto Cannella (D) https://orcid.org/0000-0002-3808-0785 Gianvincenzo Sparacia (ID https://orcid.org/0000-0002-47871634

\section{References}

1. Siskas N, Lefkopoulos A, Ioannidis I, et al. Cortical laminar necrosis in brain infarcts: serial MRI. Neuroradiology 2003; 45: 283-288.

2. Donaire A, Carreno M, Gómez B, et al. Cortical laminar necrosis related to prolonged focal status epilepticus. J Neurol Neurosurg Psychiatry 2006; 77: 104-106.

3. Lee BW, Jin ES, Hwang HS, et al. A case of hypoglycemic brain injuries with cortical laminar necrosis. J Korean Med Sci 2010; 25: 961-965.

4. Choi JM, Kim YH and Roh SY. Acute hepatic encephalopathy presenting as cortical laminar necrosis: case report. Korean J Radiol 2013; 14: 324-328.

5. Bargalló N, Burrel M, Berenguer J, et al. Cortical laminar necrosis caused by immunosuppressive therapy and chemotherapy. AJNR Am J Neuroradiol 2000; 21: 479-484.

6. Roh JH, Kim JH, Oh K, et al. Cortical laminar necrosis caused by rapidly corrected hyponatremia. J Neuroimaging 2009; 19: 185-187.

7. Arboix A, González-Peris S, Grivé E, et al. Cortical laminar necrosis related to migrainous cerebral infarction. World J Clin Cases 2013; 1: 256-259.

8. Khardenavis V, Karthik DK, Kulkarni S, et al. Cortical laminar necrosis in a case of migrainous cerebral infarction. BMJ Case Rep 2018; doi:10.1136/bcr-2017-221483.

9. Komiyama M, Nakajima H, Nishikawa $M$, et al. Serial MR observation of cortical laminar necrosis caused by brain infarction. Neuroradiology 1998; 40: 771-777.
10. Yoneda $\mathrm{Y}$ and Yamamoto $\mathrm{S}$. Cerebral cortical laminar necrosis on diffusion-weighted MRI in hypoglycaemic encephalopathy. Diabet Med 2005; 22: 1098-1100.

11. McKinney AM, Teksam M, Felice R, et al. Diffusionweighted imaging in the setting of diffuse cortical laminar necrosis and hypoxic-ischemic encephalopathy. AJNR Am J Neuroradiol 2004; 25: 1659-1665.

12. Petcharunpaisan S, Ramalho J and Castillo M. Arterial spin labeling in neuroimaging. World J Radiol 2010; 2: 384-398.

13. Kizilkilic O, Albayram S, Kasapcopur O, et al. MRI findings of hypoxic cortical laminar necrosis in a child with hemolytic anemia crisis. Eur Radiol 2003; 13: L133-L137.

14. Samain JL, Haven E, Gille M, et al. Typical CT and MRI features of cortical laminar necrosis. JBR-BTR 2011; 94: 357.

15. Kesavadas C, Santhosh K, Thomas B, et al. Signal changes in cortical laminar necrosis-evidence from susceptibility-weighted magnetic resonance imaging. Neuroradiology 2009; 51: 293-298.

16. Niwa T, Aida N, Shishikura A, et al. Susceptibilityweighted imaging findings of cortical laminar necrosis in pediatric patients. AJNR Am J Neuroradiol 2008; 29: 1795-1798.

17. Boyko OB, Burger PC, Shelburne JD, et al. Non-heme mechanisms for T1 shortening: pathologic, CT, and MR elucidation. AJNR Am J Neuroradiol 1992; 13: 1439-1445.

18. Castillo M, Scatliff JH, Kwock L, et al. Postmortem MR imaging of lobar cerebral infarction with pathologic and in vivo correlation. Radiographics 1996; 16: 241-250.

19. Han SR, Yee GT, Choi CY, et al. Cortical laminar necrosis in an infant with severe traumatic brain injury. J Korean Neurosurg Soc 2011; 50: 472-474.

20. Niibo $T$, Ohta $H$, Yonenaga $K$, et al. Arterial spinlabeled perfusion imaging to predict mismatch in acute ischemic stroke. Stroke 2013; 44: 2601-2603.

21. Morais R, Sobral F, Cunha G, et al. Advanced MRI study of migrainous infarction presenting as cortical laminar necrosis - case report and literature review. Clin Neurol Neurosurg 2018; 167: 82-85.

22. Williams DS, Detre JA, Leigh JS, et al. Magnetic resonance imaging of perfusion using spin inversion of arterial water. Proc Natl Acad Sci USA 1992; 89: 212-216.

23. van Laar PJ, van der Grond J and Hendrikse J. Brain perfusion territory imaging: methods and clinical applications of selective arterial spin-labeling MR imaging. Radiology 2008; 246: 354-364.

24. Haller S, Zaharchuk G, Thomas DL, et al. Arterial spin labeling perfusion of the brain: emerging clinical applications. Radiology 2016; 281: 337-356.

25. Brumm KP, Perthen JE, Liu TT, et al. An arterial spin labeling investigation of cerebral blood flow deficits in chronic stroke survivors. Neuroimage 2010; 51: 995-1005.

26. Hartkamp NS, van Osch MJ, Kappelle J, et al. Arterial spin labeling magnetic resonance perfusion imaging in cerebral ischemia. Curr Opin Neurol 2014; 27: 42-53.

27. Huck S, Kerl HU, Al-Zghloul M, et al. Arterial spin labeling at 3.0 Tesla in subacute ischemia: comparison to dynamic susceptibility perfusion. Clin Neuroradiol 2012; 22: 29-37.

28. Guo L, Zhang Q, Ding L, et al. Pseudo-continuous arterial spin labeling quantifies cerebral blood flow in patients 
with acute ischemic stroke and chronic lacunar stroke. Clin Neurol Neurosurg 2014; 125: 229-236.

29. de Havenon A, Sultan-Qurraie A, Tirschwell D, et al. Medial occipital lobe hyperperfusion identified by arterial spin-labeling: a poor prognostic sign in patients with hypoxic-ischemic encephalopathy. AJNR Am J Neuroradiol 2015; 36: 2292-2295.

30. Yu S, Liebeskind DS, Dua S, et al. Postischemic hyperperfusion on arterial spin labeled perfusion MRI is linked to hemorrhagic transformation in stroke. J Cereb Blood Flow Metab 2015; 35: 630-637.

31. Rudilosso S, Laredo C, Mancosu M, et al. Cerebral perfusion and compensatory blood supply in patients with recent small subcortical infarcts. J Cereb Blood Flow Metab. Epub ahead of print 13 February 2018. DOI: $10.1177 / 0271678 X 18758548$ 